tions and their prognostic contribution in chronic myelomonocytic leukemia: a two-center study of 466 patients. Leukemia. 2014;28(11):2206-2212.

4. Elena C, Gallì A, Such E, et al. Integrating clinical features and genetic lesions in the risk assessment of patients with chronic myelomonocytic leukemia. Blood. 2016;128(10):1408-1417.

5. Patnaik MM, Vallapureddy R, Lasho TL, et al. EZH2 mutations in chronic myelomonocytic leukemia cluster with ASXL1 mutations and their co-occurrence is prognostically detrimental. Blood Cancer J. 2018;8(1):12

6. Patnaik MM, Barraco D, Lasho TL, et al. DNMT3A mutations are associated with inferior overall and leukemia-free survival in chronic myelomonocytic leukemia. Am J Hematol. 2017;92(1):56-61.

7. Montalban-Bravo G, Takahashi K, Patel K, et al. Impact of the number of mutations in survival and response outcomes to hypomethylating agents in patients with myelodysplastic syndromes or myelodysplastic/myeloproliferative neoplasms. Oncotarget. 2018;9(11):9714-9727.

8. Such E, Germing U, Malcovati L, et al. Development and validation of a prognostic scoring system for patients with chronic myelomonocytic leukemia. Blood. 2013;121(15):3005-3015.

9. Onida F, Kantarjian HM, Smith TL, et al. Prognostic factors and scoring systems in chronic myelomonocytic leukemia: a retrospective analysis of 213 patients. Blood. 2002;99(3):840-849.

10. Greenberg PL, Tuechler H, Schanz J, et al. Revised international prognostic scoring system for myelodysplastic syndromes. Blood. 2012;120(12):2454-2465.

11. Pleyer L, Leisch M, Kourakli A, et al. Effects of the therapeutic armamentarium on survival and time to next treatment in CMML subtypes: an international analysis of 950 cases coordinated by the AGMT study group. Blood. 2019;134(Suppl 1):844-844.
12. Park S, Labopin M, Yakoub-Agha I, et al. Allogeneic stem cell transplantation for chronic myelomonocytic leukemia: a report from the Societe Francaise de Greffe de Moelle et de Therapie Cellulaire. Eur J Haematol. 2013;90(5):355-364.

13. Symeonidis A, van Biezen A, de Wreede L, et al. Achievement of complete remission predicts outcome of allogeneic haematopoietic stem cell transplantation in patients with chronic myelomonocytic leukaemia. A study of the Chronic Malignancies Working Party of the European Group for Blood and Marrow Trans. Br J Haematol. 2015;171(2):239-246

14. Kongtim P, Popat U, Jiménez A, et al. Treatment with hypomethylating agents before allogeneic stem cell transplant improves progression-free survival for patients with chronic myelomonocytic leukemia. Biol Blood Marrow Transplant. 2016;22(1):47-53.

15. Liu HD, Ahn KW, Hu Z-H, et al. Allogeneic hematopoietic cell transplantation for adult chronic myelomonocytic leukemia. Biol Blood Marrow Transplant. 2017;23(5):767-775.

16. Sanz GF. A Lot to learn about allogeneic hematopoietic cell transplantation for chronic myelomonocytic leukemia. Biol Blood Marrow Transplant. 2017;23(5):713-714.

17. Woo J, Ro Choi D, Storer BE, et al. Impact of clinical, cytogenetic, and molecular profiles on long-term survival after transplantation in patients with chronic myelomonocytic leukemia. Haematologica. 2020;105(3):652-660

18. de Witte T, Bowen D, Robin M, et al. Allogeneic hematopoietic stem cell transplantation for MDS and CMML: recommendations from an international expert panel. Blood. 2017;129(13):1753-1762.

19. Sanz GF, Ibáñez M, Such E. Do next-generation sequencing results drive diagnostic and therapeutic decisions in MDS? Blood Adv. 2019;3(21):3454-3460.

\title{
Leukemia stem cell gene expression signatures contribute to acute myeloid leukemia risk stratification
}

\author{
Katherine L. B. Knorr and Aaron D. Goldberg
}

Division of Hematologic Malignancies, Department of Medicine, Memorial Sloan Kettering Cancer Center, New York, NY, USA

E-mail: AARON D. GOLDBERG - goldbera@mskcc.org

doi:10.3324/haematol.2019.241117

T he majority of patients with acute myeloid leukemia (AML) will die of their disease. Nevertheless, the prognosis of AML varies widely. Some AML patients may be cured by chemotherapy alone, while others require approaches such as allogeneic stem cell transplantation to have the best chance of longterm survival. As physicians, we are often asked by our AML patients: "How likely is this treatment going to work, and how long do I have to live?"1

Prognostication in AML has evolved over time. Initially, models for prediction of response to therapy were based on patient's parameters such as age and performance status in combination with cell characteristics such as morphology and chromosomal karyotype. With technological advancements, our understanding of disease biology has evolved and factors including molecular mutations and minimal residual disease have been integrated into prognostication schemes. Recently, an international expert panel on behalf of the European LeukemiaNet (ELN) published a revised version of a widely utilized prognostication scheme that categorizes AML patients into three risk groups (Favorable, Intermediate, and Adverse) based on genetic abnormalities (incorporating chromosomal karyotype and specific molecular muta- tions). ${ }^{2}$ These AML risk groups have profound clinical implications, particularly with regard to post-remission therapy for younger fit patients. In general, fit Favorablerisk AML patients who achieve a first complete remission after induction chemotherapy go on to consolidation chemotherapy with curative intent. However, even fit patients with Adverse- and Intermediate-risk AML are unlikely to be cured by chemotherapy alone, and therefore it is reasonable to consider allogeneic stem cell transplantation for Intermediate- and Adverse-risk patients upon achievement of first complete remission.

Why is AML so often resistant to chemotherapy? The biology of AML chemoresistance is complex. However, at a basic level, adverse-risk AML cells are more likely to evade conventional chemotherapeutics that target the cell cycle. It has therefore been hypothesized that one powerful driver of adverse prognosis in AML may be the properties of the leukemia stem cell (LSC), a type of cell that exhibits cell cycle quiescence, self-renewal, and chemoresistance. ${ }^{3-6}$ Although AML LSC remain challenging to isolate, assessment of AML LSC gene expression signatures has been proposed as a method to further refine prognosis - with LSC-like AML phenotypes contributing to adverse risk. 
A

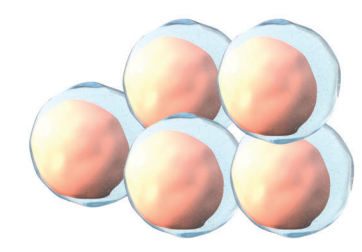

$A M L$ - 17-gene low

Biallelic CEBP $\alpha$

GATA2

KIT

$\mathbf{B}^{-}$

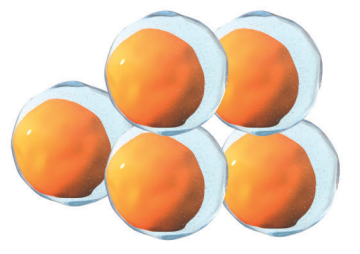

AML - 17-gene high

$\begin{array}{cc}\text { inv(3) / t(3;3) } & \text { RUNX1 } \\ \text { FLT3-ITD } & \text { SRSF2 } \\ \text { ASXL1 } & \text { STAG2 } \\ \text { DNMT3a } & \text { TET2 } \\ \text { KMT2A } & \text { TP53 }\end{array}$

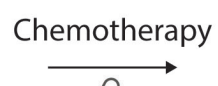

Qs

Chemotherapy

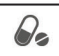

\section{Complete remission}

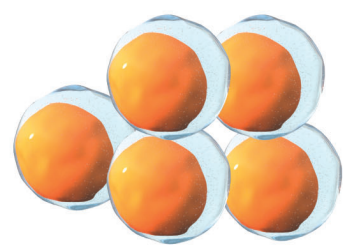

Relapsed or refractory AML

C

\section{Overall survival}

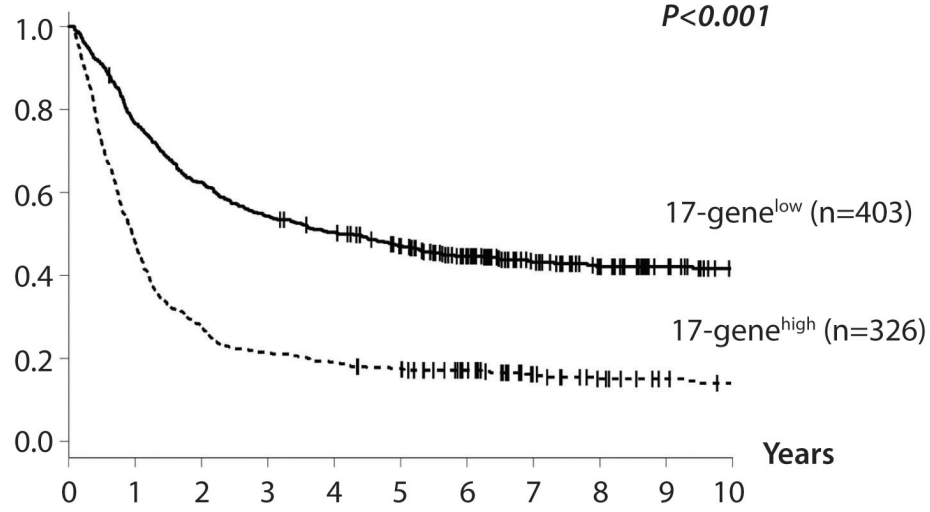

Figure 1. The 17-gene leukemia stem cell score refines prognosis in acute myeloid leukemia beyond that afforded by the European LeukemiaNet risk categories. (A) Patients with acute myeloid leukemia (AML) with a 17-gene ${ }^{\text {low }}$ leukemia stem cell (LSC) score more frequently have biallelic CEBPA, GATA2, and KIT mutations and

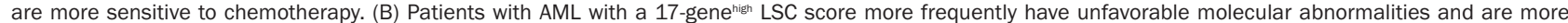
resistant to chemotherapy. (C) The 17 -gene LSC score has a powerful prognostic impact, particularly in younger adult AML patients (aged $<60$ years).

A study by $\mathrm{Ng}$ et al. recently defined a list of genes differentially expressed between LSC and non-LSC fractions (validated by xenotransplantation) from 78 AML patients. ${ }^{7}$ The list of genes highly expressed in LSC was subjected to statistical regression analysis to relate the expression profile to patients' survival, which yielded an optimal "17-gene LSC score" prognostic signature. When the scoring algorithm was applied to five cohorts of AML patients, high scores consistently correlated with poor prognostic factors such as older age, higher initial white blood cell count, and unfavorable cytogenetics. High scores also correlated with resistance to standard induc- tion chemotherapy, higher rates of relapse, and poor outcomes including inability to achieve complete response, decreased overall survival, and shorter event-free and relapse-free survival. $\mathrm{Ng}$ et al. proposed that this scoring tool could be applied to guide selection of initial therapy in newly diagnosed patients, specifically to identify highrisk patients not likely to benefit from standard induction chemotherapy.

In this issue of Haematologica, Bill et al. provide an impressive validation of the 17-gene LSC scoring system using RNA-sequencing data from a large number of patients treated in cooperative group (CALGB) trials. ${ }^{8}$ 
This work confirms and expands upon the insights published in 2016 by $\mathrm{Ng}$ et al., showing the prognostic value of LSC gene expression signatures in an independent large cohort of AML patients. Here, Bill et al. apply the 17gene LSC score to 934 de novo AML patients and report the association of the 17-gene LSC score with prognostic clinical parameters, specific AML mutations, and ELN risk classification.

Using unsorted pre-treatment bone marrow and/or peripheral blood specimens, the group conducted transcriptome analysis via RNA-sequencing. The 17-gene LSC score was calculated as the weighted sum of the normalized expression values of the 17 genes included in the signature panel defined by $\mathrm{Ng}$ et al. The scores derived were then divided into two groups using the median as

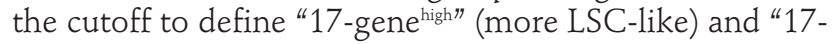
gene $^{\text {lown }}$ (less LSC-like) (Figure 1A, B).

Consistent with prior data, allocation into the 17-genehigh and 17-gene $e^{\text {low }}$ groups correlated with known prognostic factors. 17-gene ${ }^{\text {low }}$ patients were more often younger (age $<60$ years) and predominantly had favorable cytogenetic profiles. Bill et al. also correlated the 17-gene score with known AML mutations. ${ }^{8}$ Favorable mutations in genes such as CEBPA, GATA2, and KIT were more frequent in patients with a 17-gene ${ }^{\text {low }}$ score (Figure 1A) while unfavorable mutations in genes including ASXL1, RUNX1, and TP53 occurred more frequently in patients with a 17-gene $e^{\text {high }}$ score (Figure 1B). Patients with extremely high-risk EVI1 rearrangements $\operatorname{inv}(3) / \mathrm{t}(3 ; 3)$ were exclusively found in the 17 -gene engh $^{\text {hig }}$ score group. With respect to mutation burden, more LSC-like AML harbored slightly more mutations, with a median of two among patients with a 17 -gene $e^{\text {low }}$ score and of three among those with a 17-gene $e^{\text {high }}$ score.

Next, Bill et al. assessed outcomes in the groups with 17 -gene ${ }^{\text {low }}$ and 17 -gene e $^{\text {high }}$ scores. Both groups followed known associations for favorable and poor outcomes (complete remission rate, longer disease-free and overall survival) in, respectively, the younger (Figure 1C) and older cohorts of patients. In addition to validating the prognostic impact of the 17-gene LSC score in a large independent cohort and adding correlations with AML mutations, Bill et al. also compared the 17-gene LSC score to AML ELN risk stratification. ${ }^{8}$

When patients were classified according to the ELN stratification into Favorable-, Intermediate-, and Adverserisk groups, there were significant differences in ELN risk distribution between the 17-gene ${ }^{\text {low }}$ and 17 -gene $e^{\text {high }}$ LSC score patients of different ages. In younger patients with a $17-$ gene $e^{\text {low }}$ score, most $(66 \%)$ were classified as having Favorable-risk, with $14 \%$ and $17 \%$ classified as having Intermediate- and Adverse-risk, respectively. However, younger patients with a 17-gene high $^{\text {sig }}$ score were spread across the ELN classification: Adverse-risk (41\%), Intermediate-risk (32\%), and Favorable-risk $(26 \%)$. In older patients with a 17 -gene $e^{\text {low }}$ score, only $36 \%$ were classified in the Favorable-risk group, while $24 \%$ had an Intermediate risk and $40 \%$ an Adverse risk. By comparison, older patients with a 17 -gene $e^{\text {high }}$ score clustered mainly into the Adverse-risk group (63\%), with fewer in the Intermediate- (18\%), and Favorable-risk (18\%) groups.
When assessing outcomes, the 17-gene LSC score failed to add significant prognostic information to ELN classification in older AML patients, in whom prognosis remains poor across prognostic groups with conventional chemotherapy.

Intriguingly, the data suggest that the 17-gene LSC score can provide additional prognostic value particularly for younger patients who may be currently misclassified as having a favorable risk. Younger patients with an ELN Favorable-risk classification with a high 17-gene LSC score ( $20 \%$ of ELN Favorable-risk patients) have a worse prognosis than would otherwise be expected from the ELN classification alone. This unexpectedly high-risk group of patients epitomizes the rationale for using refined prognostication schemes such as the 17-gene scoring tool, with the goal of tailoring first-line therapy more precisely and identifying populations of patients in need of prospective clinical trials.

The comprehensive RNA-sequencing approach described by Bill et al. does have some limitations. From a practical point of view, while pre-treatment cytogenetics as well as genomic profiling for mutations in specific genes have become standards of care for patients with AML, it is premature to recommend universal pre-treatment RNA-sequencing. Future studies in adult AML may validate the prognostic significance of pre-treatment profiling of a limited list of LSC-related genes using more targeted gene expression analysis, as was recently shown using Nanostring technology in pediatric AML.

In a broader perspective, prognosis in any disease is shaped by the efficacy of available therapy. All patients evaluated in the current study by Bill et al. received cytarabine/anthracycline-based induction chemotherapy. ${ }^{8}$ Although AML prognosis has traditionally been evaluated in response to cytotoxic chemotherapy, the prognostic impact of ELN genetic risk classification and LSC gene expression signatures will need to be reevaluated in the context of novel and more targeted therapeutics.

Recently, the BCL-2 inhibitor venetoclax in combination with hypomethylating agents has become a new standard of care for adult patients with AML who are unfit, by virtue of age or comorbidities, to receive intensive chemotherapy. ${ }^{10}$ Although many patients still relapse, this combination shows activity in disease often refractory to standard induction chemotherapy, including secondary AML, therapy-related AML, and AML with high-risk cytogenetic and mutation profiles. One explanation for the relatively mutation-agnostic efficacy of venetoclax + azacitidine is the combination's suppression of oxidative phosphorylation and disruption of energy metabolism in LSC. ${ }^{11}$ The impact of LSC gene expression signatures on prognosis in patients treated with hypomethylating agents + venetoclax has yet to be determined. Similarly, the impact of LSC gene expression signatures on prognosis in FLT3-mutated patients may also need to be re-evaluated, as more effective and specific FLT3 inhibitors enter clinical practice. ${ }^{12}$ In general, as more effective therapies are developed that target the fundamental biology of AML, prognostic factors and even post-remission therapies will need to be re-examined. 


\section{References}

1. Liesveld J. Management of AML: who do we really cure? Leuk Res. 2012;36(12):1475-1480.

2. Dohner H, Estey E, Grimwade D, et al. Diagnosis and management of AML in adults: 2017 ELN recommendations from an international expert panel. Blood. 2017;129(4):424-447.

3. Thomas D, Majeti R. Biology and relevance of human acute myeloid leukemia stem cells. Blood. 2017;129(12):1577-1585

4. Lapidot T, Sirard C, Vormoor J, et al. A cell initiating human acute myeloid leukaemia after transplantation into SCID mice. Nature. 1994;367(6464):645-648.

5. Shlush LI, Zandi S, Mitchell A, et al. Identification of pre-leukaemic haematopoietic stem cells in acute leukaemia. Nature. 2014;506(7488):328-333

6. Pollyea DA, Gutman JA, Gore L, Smith CA, Jordan CT. Targeting acute myeloid leukemia stem cells: a review and principles for the development of clinical trials. Haematologica. 2014;99(8):1277-1284.

7. Ng SW, Mitchell A, Kennedy JA, et al. A 17-gene stemness score for rapid determination of risk in acute leukaemia. Nature. 2016;540(7633):433-437

8. Bill M, Nicolet D, Kohlschmidt J, et al. Mutations associated with a 17-gene leukemia stem cell score and the score's prognostic relevance in the context of the European LeukemiaNet classification for acute myeloid leukemia. Haematologica.2020;105(3):721-729.

9. Duployez N, Marceau-Renaut A, Villenet C, et al. The stem cellassociated gene expression signature allows risk stratification in pediatric acute myeloid leukemia. Leukemia. 2019;33(2):348-357.

10. DiNardo CD, Pratz K, Pullarkat V, et al. Venetoclax combined with decitabine or azacitidine in treatment-naive, elderly patients with acute myeloid leukemia. Blood. 2019;133(1):7-17.

11. Pollyea DA, Stevens BM, Jones CL, et al. Venetoclax with azacitidine disrupts energy metabolism and targets leukemia stem cells in patients with acute myeloid leukemia. Nat Med. 2018;24(12):18591866.

12. Perl AE, Martinelli G, Cortes JE, et al. Gilteritinib or chemotherapy for relapsed or refractory FLT3-mutated AML. N Engl J Med. 2019;381(18):1728-1740.

\title{
Thrombopoietin receptor agonists for the treatment of inherited thrombocytopenia
}

\author{
Michael Makris ${ }^{1,2}$ \\ ${ }^{1}$ Department of Infection, Immunity and Cardiovascular disease, University of Sheffield, and ${ }^{2}$ Sheffield Haemophilia and Thrombosis \\ Centre, Royal Hallamshire Hospital, Sheffield, UK \\ E-mail:m.makris@sheffield.ac.uk
}

doi:10.3324/haematol.2019.241786

T he inherited thrombocytopenias are a heterogeneous group of increasingly recognized disorders, which can be associated with bleeding of variable severity. Their prevalence has been estimated to be around 1 in 100,000 of the population, ${ }^{1}$ but it is likely that this is an underestimate due to many individuals being undiagnosed, wrongly diagnosed or not recorded on registries after a correct diagnosis. More recently, it has been reported that the prevalence of MYH9-related disorders can be as frequent as 1 in 20,000 of the population. ${ }^{2}$

The inherited nature of the thrombocytopenias has been recognized for decades, with the main disorders being the May-Hegglin anomaly, and the Sebastien, Fechtner and Epstein syndromes. These disorders were associated with a variable degree of renal impairment, deafness and cataracts. Although initially believed to be different disorders, when the genes responsible were identified, it became clear that all of these syndromes were variants of defects in the same $\mathrm{MYH} 9$ gene encoding for non-muscle myosin heavy chain $\mathrm{A} .{ }^{3}$ The nomenclature was subsequently changed to reflect this, and they are now known as the MYH9-related disorders (MYH9-RD).

The recent introduction of high throughput sequencing (HTS), together with the formation of consortia with large numbers of clinicians caring for inherited thrombocytopenia patients, has led to a dramatic increase in the number of genes responsible for the disorder. Inherited thrombocytopenias can be syndromic, predisposing to renal failure, hearing loss and cataracts, as in MYH9-RD, while others, such as the RUNX1, ANKRD26 and ETV6, can be associated with predisposition to hematologic malignancy, 4,5

In contrast to the major advances in the genetic basis of inherited thrombocytopenia, the management of these disorders has hardly changed, with the main therapeutic decision being whether to transfuse platelets or not. Part of the difficulty is the variability in the number of platelets, as well as the bleeding tendency which is often not directly proportional to the platelet count. A possible explanation for this is the variable and often large size of the platelets in some of these disorders; since hemostatic reactions take place on the cell surface, disorders associated with larger platelets would be expected to be associated with less bleeding. Treatment is usually required when patients are actively bleeding, or to prevent bleeding prior to surgery or invasive procedures.

Platelet transfusions, however, can be problematic because of the potential for adverse events. They carry the risk of transfusion-transmitted infection, alloimmunization with production of platelet specific or HLA antibodies, allergic reactions and transfusion-related acute lung injury (TRALI). As a result, the use of platelet transfusions tends to be avoided if possible, and clinicians use tranexamic acid, sometimes with desmopressin, as nonspecific hemostatic agents to treat these patients.

Thrombopoietin receptor agonists have been available for the treatment of immune thrombocytopenia in adults and children for some time. The two products with the longest availability are eltrombopag, which is given orally, and romiplostim, which is administered subcutaneously. In the UK, eltrombopag is available for use in patients with thrombocytopenia of at least six months duration whilst romiplostim is approved for ITP of 12 months duration or more.

In an important initial publication from 2010, Pecci et al. showed that eltrombopag could increase the platelet count of patients with $M Y H 9$-related thrombocytopenia. ${ }^{\circ}$ Twelve patients with a platelet count of $<50 \times 10^{\circ} / \mathrm{L}$ were 